\title{
Microbiome Profile in Adult Balanoposthitis Patients: Relationship with Redundant Prepuce, Skin Physical Barrier Status and Inflammation
}

\section{Meng Li}

China Medical University

Jian-Xun Mao

China Medical University

Hang-Hang Jiang

China Medical University

Chun-Ming Huang

University of California San Diego

Xinghua Gao ( $\nabla$ gaobarry@hotmail.com )

First Hospital of China Medical University https://orcid.org/0000-0001-8809-8564

\section{Li Zhang}

China Medical University

\section{Research}

Keywords: Balanoposthitis, Skin microbiome, Skin physical barrier, Redundant Prepuce

Posted Date: August 27th, 2020

DOI: https://doi.org/10.21203/rs.3.rs-64050/v1

License: (c) (i) This work is licensed under a Creative Commons Attribution 4.0 International License.

Read Full License 


\section{Abstract}

Background. Balanoposthitis is a common inflammatory condition of male genitalia, the microbiota spectrum have not been determined thus far. To profile the microbiome in patients with balanoposthitis (BP), and healthy controls ( $\mathrm{HC})$, and investigate its correlation with redundant prepuce, severity of inflammation, and disrupted skin barrier function, including skin $\mathrm{pH}$, Transepidermal Water Loss (TEWL), and skin hydration.

Results. In general, the microbiota between $\mathrm{BP}$ and $\mathrm{HC}$ were similar. The microbiota composition was different between BP and HC with redundant prepuce. Staphylococcus warneri and Prevotella bivia are two most abundant BP-associated Species, and positively correlated with disease severity. The physiobarrier function is markedly impaired in BP, shown by decreased skin hydration, while increased TEWL and $\mathrm{pH}$.

Conclusions. Staphylococcus warneri and Prevotella bivia showed a strong correlation with balanoposthitis and may be pathogenic for the condition.

\section{Background}

Balanoposthitis represents one of a group of the most common male genital inflammatory diseases. Inflammation involving the glans penis is referred to as balanitis, while that involving the prepuce is posthitis, and that involving both areas is referred to balanoposthitis[1]. It is believed that balanoposthitis may be caused by or associated with some disorders such as infection, inflammatory dermatosis, tumors, trauma, autoimmune diseases, and drug eruptions[2]. In addition to the contribution of Candida albicans, bacterial colonization, such as Staphylococcus aureus, group B streptococci (GBS), and group D streptococci (GDS) [3, 4], as well as anaerobic strains[5], are considered pathogenic in balanoposthitis based on conventional culture-based methods. Recent studies have revealed that the composition of the bacterial community of penile skin is quite diverse, and can be influenced by circumcision[6] and the vaginal status of their monogamous sex partners[7]. For example, there is a significant decrease in putative anaerobic bacterial families after circumcision, notably the Clostridiales and Prevotellaceae families $[6,8,9]$. It has been reported that the incidence of balanitis is $4 \%$ in children, $11 \%-13 \%$ in adults without circumcision, and $2 \%$ in adults with circumcision[10].

Thus far, the microbiota profile in balanoposthitis has yet to be established. In this report, we designed a case-controlled study to investigate the diversity of skin microbiome in patients with balanoposthitis by targeting region $\mathrm{V} 4$ of $16 \mathrm{~S}$ ribosomal RNA (rRNA) and evaluated the correlation between cutaneous microbiota with clinical features of balanoposthitis.

\section{Method}

\section{Study Participants}


The clinical materials were collected at the Department of Dermatology, the First Hospital of China Medical University, China, over the period from July 1 to November 1, 2018. This study was approved by the Ethics Committee of the First Hospital of China Medical University, Shenyang, China. (Chinese Clinical Trial Registry, Number: ChiCTR1800016901)

Inclusion and Exclusion Criteria: Patients were at the ages of 18-65 years. A clinical diagnosis of BP was given as ill-defined edematous erythematous patches, erosions, papules, and excessive smegma and that the patients experience a foul odor, soreness, or itching, based upon the 2013 European guideline for the management of balanoposthitis[2]. Patients with written informed consent. Excluded were those who received systemic or topical therapy within 1 month prior to enrollment in the study, those with severe systemic conditions and other defined genital diseases within 3 months, including but not limited to warts, psoriasis, lichens sclerosus, eczema/dermatitis and those with overt candidal infection with positive $\mathrm{KOH}$ microscopic detection showing pseudohyphae or germinative spores. Healthy controls were volunteers from university campus and employees from a company, who consented to participate, free of systemic or genital illnesses by a physician examination.

Thirty BP were screened, among them, four were excluded due to positive microscopic fungal detection. Of the thirty $\mathrm{HC}$ screened, one was excluded due to self-report indisposed condition. In total, 26 patients diagnosed with balanoposthitis (BP) and, 29 healthy controls (HC) were consecutively entered the study. Demographic data from all participants were summarized in Table 1. 
Table 1

General Characteristics and Skin Physio-Barrier Status of Participants

\begin{tabular}{|c|c|c|c|}
\hline & Balanoposthitis & Healthy Controls & p-value* \\
\hline Numbers & 26 & 29 & \\
\hline Age, mean (SD) & $36.05(11.12)$ & $40.69(12.83)$ & ns \\
\hline Married, n (\%) & $16(61.5)$ & $19(65.5)$ & ns \\
\hline BMI, mean (SD) & $24.61(4.36)$ & $25.01(3.61)$ & ns \\
\hline Redundant prepuce*, n (\%) & $25(96.1)$ & $20(69.0)$ & प \\
\hline History of STD (Total, \%) & $10(38.46)$ & $0(0)$ & - \\
\hline condyloma acuminatum & $3(11.54)$ & $0(0)$ & - \\
\hline nongonococcal urethritis & $4(15.38)$ & $0(0)$ & - \\
\hline syphilis & $1(3.85)$ & $0(0)$ & - \\
\hline genital herpes & $2(7.69)$ & $0(0)$ & - \\
\hline Condom use in sexual behavior*, $n / n$ & $8 / 17$ & $2 / 17$ & प \\
\hline Cleaning Frequency, n/d(SD) & $0.98(0.49)$ & $0.72(0.40)$ & ns \\
\hline $\mathrm{pH}$, mean (SD) & $6.34(0.86)$ & $5.71(0.42)$ & प \\
\hline In HCNP, mean (SD) & & $5.71(0.40)$ & प \\
\hline In HCRP, mean (SD) & & $5.71(0.43)$ & प \\
\hline TEWL, mean (SD) g/hm² & $55.52(12.69)$ & $47.03(11.77)$ & प \\
\hline In HCNP, mean (SD) & & $40.36(12.08)$ & $\mathrm{Qu}$ \\
\hline In HCRP, mean (SD) & & $50.04(10.59)$ & ns \\
\hline Skin hydration, mean (SD) & $66.62(24.68)$ & $81.30(19.36)$ & प \\
\hline In HCNP, mean (SD) & & $66.62(11.35)$ & ns \\
\hline In HCRP, mean (SD) & & $91.46(12.18)$ & वस्पा \\
\hline \multicolumn{4}{|c|}{$\begin{array}{l}\text { Abbreviations: BMI, body mass index; SD, standard deviation; STD, sexually transmitted diseases; d, } \\
\text { day; pH, logarithm of hydrogen ion concentration reciprocal; TEWL, transepidermal water loss; HCNP, } \\
\text { healthy control with normal prepuce; HCRP, healthy control with redundant prepuce. }\end{array}$} \\
\hline \multicolumn{4}{|c|}{ p-value*: ns, non-significant difference; $\square p<0.05,0 p<0.005,0<0.0005$} \\
\hline \multicolumn{4}{|c|}{$\begin{array}{l}\text { Redundant prepuce* denotes foreskins that completely cover the glans during erection but retain the } \\
\text { capacity to retract manually. }\end{array}$} \\
\hline
\end{tabular}


Sampling for microbiome detection, clinical assessment and, measurement of physical barrier parameters

Participants were instructed to retract and flatten the foreskin. The glans penis and prepuce were scrubbed with a sterile cotton swab. Samples were placed in sealed sterile containers, transported to the laboratory and stored at $-80^{\circ} \mathrm{C}$. At 30-min after sampling, two dermatologists graded symptom severity according to a 4-grade score ( $0-3$ points) and arrived at a consensus. Items included within this severity score scale included erythema, edema, erosions/ulcers, papules/blisters/pustules, smegma, itching, pain, and foul odor (Table 2). Physio-barrier properties of the ventral surface of the glans penis, including skin $\mathrm{pH}$, trans-epidermal water loss (TEWL), and hydration content, were measured using the Courage + Khazaka electronic GmbH MPA 10 (Courage Khazaka, Version: 2.3.4.1/2017/2/1) as instructed by the manual. Measurement for each site was repeated three times.

Table 2

Severity Scores of Balanoposthitis Patients

\begin{tabular}{|c|c|c|c|c|}
\hline Score & 0 & 1 & 2 & 3 \\
\hline $\begin{array}{l}\text { Extent of } \\
\text { involvement }\end{array}$ & None & Penis crown & $\begin{array}{l}\text { Either glans or inner } \\
\text { prepuce }\end{array}$ & $\begin{array}{l}\text { Whole glans and } \\
\text { inner prepuce }\end{array}$ \\
\hline Erythema degree & No & Mildly reddish & Fresh red with edema & $\begin{array}{l}\text { Fiery red with } \\
\text { apparent edema }\end{array}$ \\
\hline Erosion/ulcers area* & 0 & $30 \%$ & $30-60 \%$ & $>60 \%$ \\
\hline $\begin{array}{l}\text { Number of papules/ } \\
\text { blisters / pustules }\end{array}$ & No & $1-9$ & $10-30$ & $>30$ \\
\hline Smegma & No & $\begin{array}{l}\text { Visible smegma } \\
\text { upon cotton } \\
\text { swabbing }\end{array}$ & Visible smegma & Dense smegma \\
\hline Foul odor & No & Slight odor & Easily detected odor & Strong odor \\
\hline Itching & No & Mild itching & $\begin{array}{l}\text { Considerable itching } \\
\text { producing irritation }\end{array}$ & $\begin{array}{l}\text { Severe itching } \\
\text { even affecting } \\
\text { sleep }\end{array}$ \\
\hline Pain & No & Mildly painful & $\begin{array}{l}\text { Considerable pain } \\
\text { producing irritation }\end{array}$ & $\begin{array}{l}\text { Severe pain even } \\
\text { affecting sleep }\end{array}$ \\
\hline
\end{tabular}

\section{DNA extraction and Sequence processing}

Total genomic DNA was extracted by using the CTAB (hexadecyltrimethylammonium bromide) method. The V4 region of the $16 \mathrm{~S}$ rRNA was amplified by polymerase chain reaction using primers $515 \mathrm{~F} / 806 \mathrm{R}$ as described[11]. Amplicons were sequenced on a desktop sequencer (Ion S5 XL, Thermo Scientific, Waltham, MA). Soft Cutadapt was used to filter low abundance reads and short read lengths, while 
Operational Taxonomic Units (OTUs) were clustered and species annotated based on $a \geq 97 \%$ nucleotide sequence identity[12].

\section{Statistical Analysis}

Statistical analysis and graphic visualizations were performed using soft R (version 3.5.2). Differences in skin physical barrier function items between the groups were compared by Student's t-test. Community richness index (the observed species) and diversity index (the Shannon Wiener index) were calculated with use of the phyloseq package on raw data for Alpha diversity, and statistically tested using the Wilcoxon rank-sum test, the correlation between Alpha diversity and Severity Scores was analyzed using linear regression. For Beta diversity (microbial compositions), community structure differences between the groups were visualized with principal coordinate analysis (PCoA) plots based on Weighted UniFrac distances, tested by Multi Response Permutation Procedure (MRPP) based on Bray-Curtis dissimilarity index. Linear Discriminant Analysis Effect Size (LEfSe) was used to determine whether a particular OTUs abundance was statistically different between groups. Random Forest (R packages "randomForest") was used to analyze the associations between the top 35 OTUs and Severity Scores, and skin physio-barrier. The level of statistical significance was set at $a=0.05$.

\section{Results}

\section{Clinical Features of Participants}

As shown in Table 1, BP and $\mathrm{HC}$ cohorts were comparable concerning age, marital status, cleansing frequencies, and BMI (all $p>0.05$ ). There were more participants with redundant prepuce in BP patients than the HCs $(25 / 26$ vs $20 / 29, p<0.05)$. BP patients had higher rates with STI history $(10 / 26$ vs $0 / 29, p<$ $0.05)$ and condom use $(8 / 17$ vs $2 / 17, p<0.05)$ during sexual intercourse than the HCs. We developed a preliminary scoring algorithm to reflect the severity of the disease. The major clinical signs and symptoms were ranked and scored, following the principles that of scoring eczema (EASI). The scores ranged from 0-24, as shown in Table 2. 26 patients were scored with an average score of 7.08.

\section{Microbial distribution of BP and $\mathrm{HC}$}

A total of 4,429,670 paired-end reads were obtained from all samples. After sequence assembly, quality filtering, and chimera removal, a dataset of 4,192,347 high-quality, classifiable 16S rRNA gene sequences with an average of 76,224 sequences per sample were obtained. The cutaneous microbiome was characterized based on the Silva132 Database. OTUs $(8,963)$ were annotated with taxonomic information related to 63 bacterial Phyla, 81 Classes, 179 Orders, 347 Families, 1,054 Genera, and 919 Species. The most dominant Phyla in both groups were Actinobacteria, Proteobacteria, Firmicutes, and Bacteroidetes. The top 10 Genera with the greatest relative abundance in both groups were Finegoldia, Stenotrophomonas, Lactobacillus, unidentified Corynebacteriaceae, unidentified Prevotellaceae, Porphyromonas, Prevotella, Ezakiella, Dialister, and Staphylococcus. 


\section{Inter- and Intra-group Differences of Skin Microbiome between $\mathrm{BP}$ and $\mathrm{HC}$}

There were no statistically significant differences in Alpha diversity between the BP and HC groups ( $p$ > 0.05), by community richness index (the observed species) and diversity index (the Shannon Wiener index) analysis, albeit species diversity levels within 43 out of 55 samples were high (mean of Shannon indexes were $>4)$. The observed species of BP were inversely correlated with disease severity scores $(\mathrm{p}<$ 0.05), while Shannon Wiener index was not correlated with disease severity.

Next, we analyzed microbiome composition (Beta diversity) by MRPP and displayed by PCoA. The differences in microbiome composition between the BP and HC groups were not significant $(p=0.11$ > 0.05, MRPP). Prepuce redundancy is considered to be an important factor in the onset and development of balanoposthitis and circumcision renders a good prognosis [13]. All but one of the BP had redundant prepuce, while nine $\mathrm{HC}$ had normal prepuce (HCNP) and twenty HC had redundant prepuce (HCRP). Beta diversity were statistically different between HCNP and HCRP, BP and HCRP (all $p<0.05$ ), but no statistical difference between BP and HCNP ( $p>0.05)$, see Fig. 1. These results suggested that both redundant prepuce and inflammation might affect skin microbiome composition. Again, there was no statistically significant differences in the Shannon index and observed species among the BP group, HCNP and HCRP subgroups.

\section{Skin Physical Barrier Status of Participants}

TEWL, skin hydrational state, and $\mathrm{pH}$ reflect the physio-barrier properties of the skin. The TEWL and $\mathrm{pH}$ were statistically significantly increased in BP as compared to $\mathrm{HC}$, that of hydration content was significantly decreased in BP as compared to HC. When the length of prepuce was counted, HCRP subjects had significantly increased TEWL, decreased water content, as compared to HCNP, while the pH remained similar (Table 1). The pH of BP (6.34) was higher than that of HCNP (5.71) and HCRP (5.71). $\mathrm{BP}$ was more alkaline than that of $\mathrm{HC}$ and exceeded the normal range of 4.1-5.8[14].

Staphylococcus warneri and Prevotella bivia are two balanoposthitis-associated Species

To determine different OTUs among groups, LDA $>4$ were chosen as assessed with the LEfSe.

Staphylococcus warneri and Prevotella bivia were identified as two major species in BP group compared to HC group (Fig. 2, upper panel). When compared with HCNP and HCRP subgroups, again, Staphylococcus warneri and Prevotella bivia were the major species in BP (Fig. 2, lower panel). Species Prevotella bivia was abundant in unmarried BP $(\operatorname{LDA}=4.513, \mathrm{p}<0.05)$, Species Staphylococcus warneri was abundant in BP with condom use (LDA $=4.420, p<0.05)$. Besides, Genus Phyllobacterium was enriched in BP group, especially married (LDA $=4.094, \mathrm{p}<0.05)$. Genus Ezakiella was enriched in HCRP subgroup, Species Porphyromonas somerae was enriched in HCNP subgroup. 


\section{Relationship Between the Microbiome and Severity of Balanoposthitis, and skin physio-barrier function}

We selected the abundant OTUs defined by the sum number of per sample reads that exceeded 10,000 (uniform treatment to 40,289 reads per sample). Top 35 abundant OTUs from the 8,963 OTUs were selected. A relationship between the microbial composition of the top 35 OTUs and severity scores was analyzed by using the Gini index of Random Forest. The abundance of OTUs served as the independent variable while severity score represented as the value of each sample as continuous dependent variables. We found that the Species Prevotella bivia, Staphylococcus warneri, and Genus Phyllobacterium were prevalent in cases with higher severity scores.

(Fig. 3). The same method was used to analyze the correlation between microbiota and skin physiobarrier function. Genus Phyllobacterium, Genus Dialister, Species Prevotella corporis, Genus unidentified Cyanobacteria, and Genus unidentified Corynebacteriaceae were the first five OTUs related to high pH. Genus Stenotrophomonas, Species Bacteroides fragilis, Genus Stenotrophomonas. 1, Species Corynebacterium tuberculostearicum, and Species Enterococcus durans were the first five OTUs related to high TEWL. Genus Phyllobacterium, Genus Ezakiella, Species Negativicoccus succinicivorans, Genus unidentified Corynebacteriaceae and Species Bacteroides fragilis were the first five OTUs related to high skin hydration. (data not shown)

\section{Discussion}

Balanoposthitis is a common inflammatory skin disease that mostly affects uncircumcised males. The diagnostic criteria for this relatively common condition, remain vague and depend mainly on clinical manifestations and exclusion of overt infection, along with specific inflammatory and neoplastic skin diseases. The main purpose of histopathological examination is to confirm the special type of balanoposthitis such as circinate balanitis and plasma cell balanitis, and to exclude pre-malignant conditions[2]. When accompanied by persistent erythematous lesions involving the balanopreputial sac in uncircumcised patients, infiltration of lymphocytes, histiocytes, and plasma cells can be seen in upper part of the chorion[15].

The cutaneous microbiome can be influenced by physio-barrier integrity, inflammation, antimicrobial peptides, and even lipids, as reported in atopic dermatitis[16, 17]. The thin epithelium of prepuce and glans penis have similarities to other parts of the skin, except that there was no hair follicle or eccrine sweat gland exist on glans penis, penis crown, and preputial mucosa[18]. The BP enrolled in the present study showed a higher rate of the redundant prepuce (26 cases but 1 ), higher incidences of STIs, and tended to use condom during sexual behavior. Indeed, bacteria enriched in BP were influenced by these factors, like Genus Phyllobacterium, Prevotella bivia, and Staphylococcus warneri.

In the present study, we profile the bacterial diversity and richness in BP by using a high throughput $16 \mathrm{~s}$ rRNA sequencing and to compare the physio-barrier status between BP and $\mathrm{HC}$ and score their 
inflammation severity by designing a scoring algorithm for balanoposthitis. To the best of our knowledge, this is the first report to assess the skin microbiome as related to balanoposthitis and skin physical barrier in the glans penis.

In regard to physio-barrier status, we found that both BP and HCRP similarly had increased TEWL and skin hydration, which maintained a higher level of water content and exudation compared to HCNP. The high $\mathrm{pH}$ value, as well as a higher level of water content in BP, may favor the growth of certain bacteria, like that of the optimal pH of 7.5 for Staphylococcus aureus[14]. It has been reported that cutaneous $\mathrm{pH}$ is influenced by filaggrin degradation, fatty acid content, sodium-hydrogen exchanger (NHE1) activation, melanosome release[14]. In the present study, we found that there was no difference in pH between healthy subjects with normal or redundant prepuce.

We could not find significant difference in Alpha or Beta diversity between BP and $\mathrm{HC}$, in general. We confirmed the previous finding that there was a significant difference in Beta diversity between HCNP and HCRP, suggesting that the prepuce could not be a neglected niche for local anatomical environment[9]. Noting that there was a significant difference in Beta diversity between BP and HCRP, we postulate that inflammatory status is more prevailing to determine the Beta diversity. Specifically, we found the observed species decreased with higher severity scores of BP, similar findings were reported in atopic dermatitis that Eczema Area and Severity Index negatively correlated with the observed species[19].

By LEfSe analysis, we found that Staphylococcus warneri and Prevotella bivia were two balanoposthitisassociated species, which were more abundant in patients with more disease severity. Staphylococcus warneri is a coagulase-negative pathogen capable of producing several virulent factors, such that factors with ability to adhere and invade human epithelial cells, to form biofilm via ica-independent mechanisms, and to destroy cells by extracellular toxins[20]. Moreover, Staphylococcus warneri has been shown to be involved in spontaneous abortion in cattle[21] and humans[22]. Whether BP was associated with spontaneous abortion is an interesting topic to investigate. Prevotella bivia was reported as a cause of bacterial vaginosis, through its capacity to alter barrier properties and induction of epithelial cell activation in the vagina[23]. We postulated that Prevotella bivia is pathogenic to BP. As Genus Prevotella could be found in the urethra and bladder[24], retrogradation of Prevotella bivia in BP could not be ignored.

\section{Conclusions}

In summary, we showed that the microbiome in BP could be associated with clinical, length of foreskin, physio-barrier status, and behavioral factors. Staphylococcus warneri and Prevotella bivia emerged predominantly in BP, and its pathogenic role remains to be tackled.

\section{Abbreviations}


Participants diagnosed with balanoposthitis

$\mathrm{HC}$

Healthy controls

HCNP

Healthy controls with normal prepuce

HCRP

Healthy controls with redundant prepuce

TEWL

Transepidermal Water Loss

OTUs

Operational Taxonomic Units

\section{LEfSe}

Linear Discriminant Analysis Effect Size

MRPP

Multi Response Permutation Procedure

\section{Declarations}

\section{Ethics approval and consent to participate}

This study carried out following the principles expressed in the Declaration of Helsinki was approved by the Ethics Committee of the First Hospital of China Medical University, Shenyang, China. (Chinese Clinical Trial Registry, Number: ChiCTR1800016901, Registered 1 July 2018, http://www.chictr.org.cn/searchproj.aspx)

\section{Consent for publication}

Not applicable.

\section{Availability of data and material}

Genomics datasets have been submitted to GSA Accession Number: CRA003111.

\section{Competing interests}

We have no conflicts of interest to disclose.

\section{Funding}

This work was supported by the National Natural Science Foundation of China (Grant No. 81673070) and the 111 Project of Ministry of Education (D18011).

\section{Authors' contributions}


The clinical experiments were designed and supervised by XHG and LZ, performed by ML and JXM. Skin microbial samples and bioinformation were processed by HHJ and CMH. ML and JXM wrote the manuscript, LZ and XHG revised manuscript. All authors read and approved the final manuscript. ML and JXM contributed equally to this work.

\section{Acknowledgments}

We would like to thank Prof. Mao-qiang Man of Dermatology, VAMC San Francisco, Northern California Institute for Research and Education and Prof. Yu-ping Lai of School of Life Science, East China Normal University for their critical revision of this manuscript.

\section{References}

1. English JC 3rd, Laws RA, Keough GC, Wilde JL, Foley JP, Elston DM. Dermatoses of the glans penis and prepuce. J Am Acad Dermatol. 1997;37(1):1-24. quiz 25-26.

2. Edwards SK, Bunker CB, Ziller F, van der Meijden WI. 2013 European guideline for the management of balanoposthitis. Int J STD AIDS. 2014;25(9):615-26.

3. Alsterholm M, Flytstrom I, Leifsdottir R, Faergemann J, Bergbrant IM. Frequency of bacteria, Candida and malassezia species in balanoposthitis. Acta Derm Venereol. 2008;88(4):331-6.

4. Lisboa C, Ferreira A, Resende C, Rodrigues AG. infectious balanoposthitis management, clinical and laboratory. Int J Dermatol. 2009;48(2):121-4.

5. Cree GE, Willis AT, Phillips KD, Brazier JS. Anaerobic balanoposthitis. Br Med J (Clin Res Ed). 1982;284(6319):859-60.

6. Price LB, Liu CM, Johnson KE, Aziz M, Lau MK, Bowers J, Ravel J, Keim PS, Serwadda D, Wawer MJ, et al. The effects of circumcision on the penis microbiome. PLoS One. 2010;5(1):e8422.

7. Zozaya M, Ferris MJ, Siren JD, Lillis R, Myers L, Nsuami MJ, Eren AM, Brown J, Taylor CM, Martin DH. Bacterial communities in penile skin, male urethra, and vaginas of heterosexual couples with and without bacterial vaginosis. Microbiome. 2016;4:16.

8. Nelson DE, Dong Q, Van der Pol B, Toh E, Fan B, Katz BP, Mi D, Rong R, Weinstock GM, Sodergren E, et al. Bacterial communities of the coronal sulcus and distal urethra of adolescent males. PLoS One. 2012;7(5):e36298.

9. Liu CM, Hungate BA, Tobian AA, Serwadda D, Ravel J, Lester R, Kigozi G, Aziz M, Galiwango RM, Nalugoda F, et al. Male circumcision significantly reduces prevalence and load of genital anaerobic bacteria. MBio. 2013;4(2):e00076.

10. Morris BJ, Krieger JN. Penile Inflammatory Skin Disorders and the Preventive Role of Circumcision. Int J Prev Med. 2017;8:32.

11. Caporaso JG, Lauber CL, Walters WA, Berg-Lyons D, Huntley J, Fierer N, Owens SM, Betley J, Fraser L, Bauer $\mathrm{M}$, et al. Ultra-high-throughput microbial community analysis on the Illumina HiSeq and MiSeq platforms. ISME J. 2012;6(8):1621-4. 
12. Liu Y, Qin Y, Guo XX, Bai Y. [Methods and applications for microbiome data analysis]. Yi Chuan. 2019;41(9):845-62.

13. Johnson KE, Sherman ME, Ssempiija V, Tobian AA, Zenilman JM, Duggan MA, Kigozi G, Serwadda D, Wawer MJ, Quinn TC, et al. Foreskin inflammation is associated with HIV and herpes simplex virus type-2 infections in Rakai, Uganda. AIDS. 2009;23(14):1807-15.

14. Proksch E. pH in nature, humans and skin. J Dermatol. 2018;45(9):1044-52.

15. Alessi E, Coggi A, Gianotti R. Review of 120 biopsies performed on the balanopreputial sac. from zoon's balanitis to the concept of a wider spectrum of inflammatory non-cicatricial balanoposthitis. Dermatology. 2004;208(2):120-4.

16. Fischer CL. Antimicrobial Activity of Host-Derived Lipids. Antibiotics (Basel) 2020, 9(2).

17. Baurecht $H$, Ruhlemann MC, Rodriguez E, Thielking F, Harder I, Erkens AS, Stolzl D, Ellinghaus $E$, Hotze M, Lieb W, et al. Epidermal lipid composition, barrier integrity, and eczematous inflammation are associated with skin microbiome configuration. J Allergy Clin Immunol. 2018;141(5):1668-76 e1616.

18. James WD, Elston D, Berger T: Andrew's Diseases of the Skin E-Book: Elsevier Health Sciences; 2011.

19. Gonzalez ME, Schaffer JV, Orlow SJ, Gao Z, Li H, Alekseyenko AV, Blaser MJ. Cutaneous microbiome effects of fluticasone propionate cream and adjunctive bleach baths in childhood atopic dermatitis. J Am Acad Dermatol. 2016;75(3):481-93 e488.

20. Szczuka E, Krzyminska S, Kaznowski A. Clonality, virulence and the occurrence of genes encoding antibiotic resistance among Staphylococcus warneri isolates from bloodstream infections. J Med Microbiol. 2016;65(8):828-36.

21. Barigye R, Schaan L, Gibbs PS, Schamber E, Dyer NW. Diagnostic evidence of Staphylococcus warneri as a possible cause of bovine abortion. J Vet Diagn Invest. 2007;19(6):694-6.

22. Waites KB, Bobo RA, Davis RO, Brookings ES, Cassell GH. Clinically silent polymicrobial amnionitis and intrauterine fetal death associated with a Cu-7 intrauterine contraceptive device. Am J Obstet Gynecol. 1984;150(8):998-9.

23. Doerflinger SY, Throop AL, Herbst-Kralovetz MM. Bacteria in the vaginal microbiome alter the innate immune response and barrier properties of the human vaginal epithelia in a species-specific manner. J Infect Dis. 2014;209(12):1989-99.

24. Gottschick C, Deng ZL, Vital M, Masur C, Abels C, Pieper DH, Wagner-Dobler I. The urinary microbiota of men and women and its changes in women during bacterial vaginosis and antibiotic treatment. Microbiome. 2017;5(1):99.

\section{Figures}




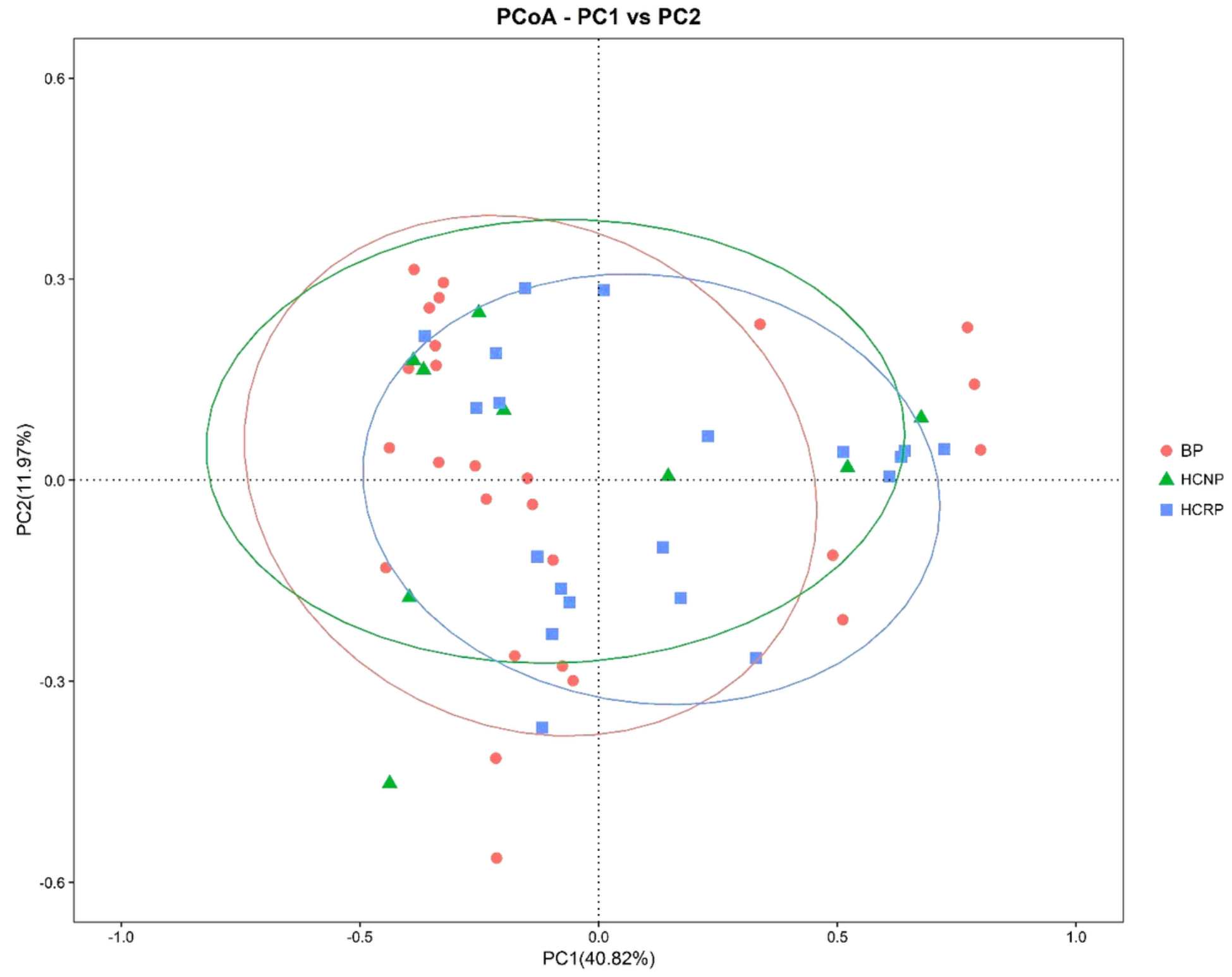

Figure 1

Column Diagrams of Skin barrier function. Principal coordinate analysis (PCoA) plots based on weighted UniFrac distances. The $x$ - and $y$-axes of PCoA indicate the first and second coordinates, respectively. BPHCNP-HCRP (HCNP-HCRP, $p<0.05$; BP-HCRP, $p<0.05$, BP-HCNP, $p>0.05$; MRPP). 

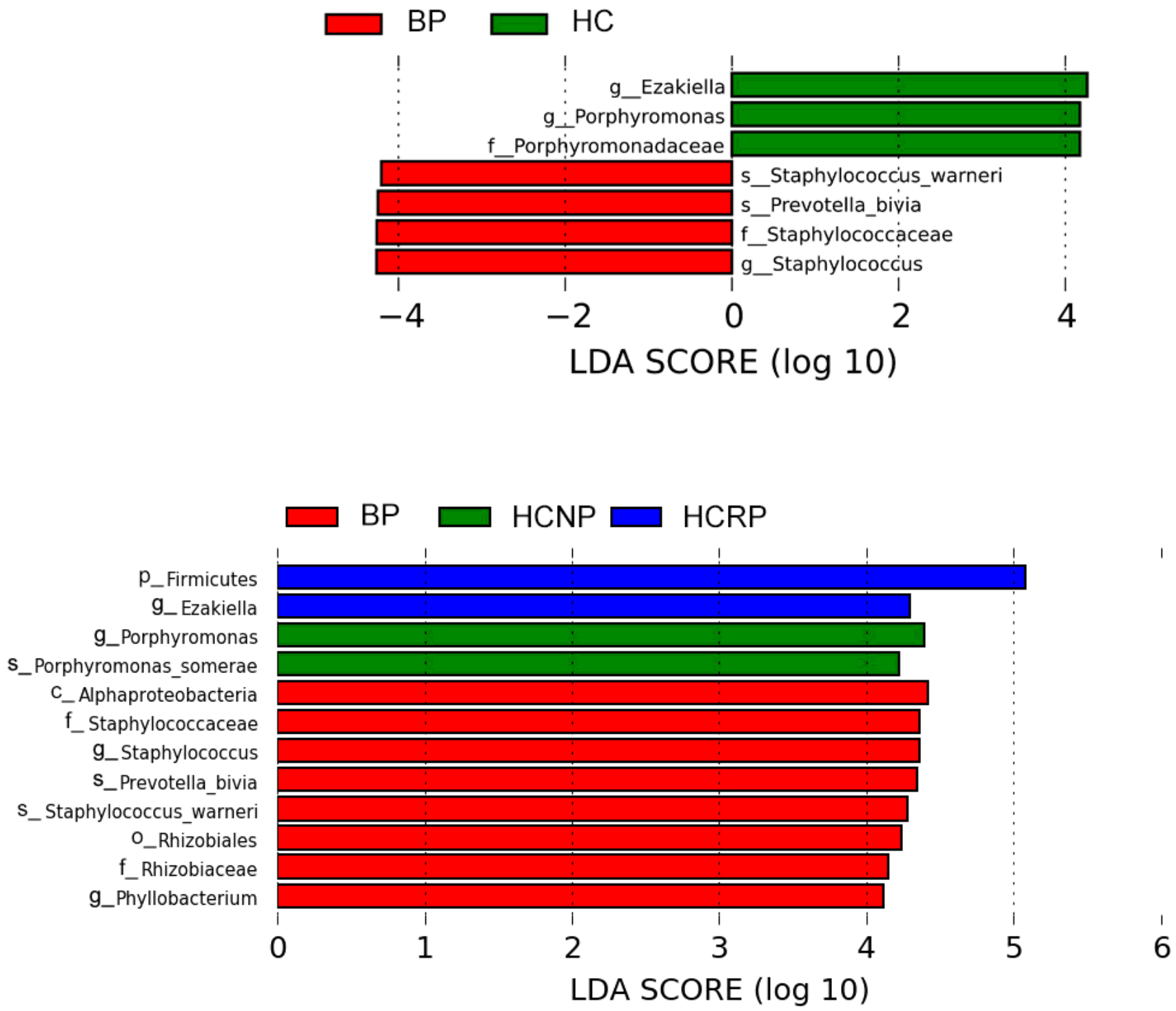

\section{Figure 2}

LEfSe analysis of significantly different OTUs between groups OTUs with significant differences among groups are shown, results with LDA $>4$ were selected. Taxonomic levels are represented with prefix $p_{-}$: Phylum, c_: Class, o_: Order, f_: Family, g_: Genus, s_: Species. 


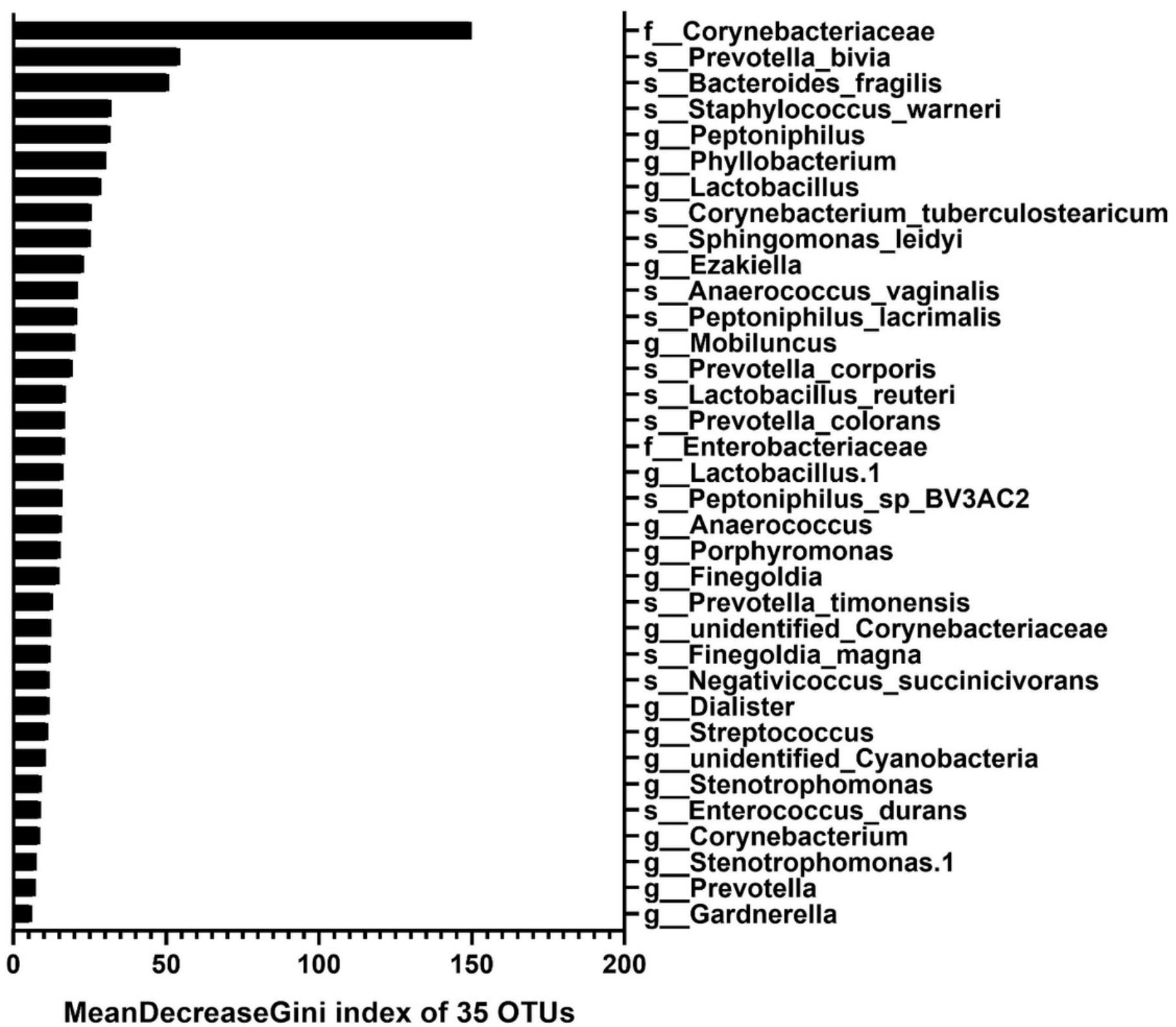

Figure 3

MeanDecreaseGini index of 35 OTUs and Severity Scores. Gini index of OTUs using Random Forest to analyze associations between the 35 abundant OTUs and Severity Scores. The larger value of MeanDecreaseGini, the greater the importance of the OTUs for severity scores. Taxonomic levels are represented with prefix f_: Family, g_: Genus, s_: Species. 